\title{
THE GENERAL INNER-OUTER FACTORIZATION PROBLEM FOR DISCRETE-TIME SYSTEMS
}

\author{
C. Oară $\breve{A}^{\star}$ A. Varga ${ }^{\star \star}$ \\ ^Faculty of Automatic Control and Computers \\ University Polytechnica Bucharest \\ Austrului 34, RO-73115, Bucharest, Romania \\ Fax: +(40) 13 234 234; Email: oara@popov.riccati.pub.ro \\ ${ }^{\star \star}$ German Aerospace Center, DLR - Oberpfaffenhofen \\ Institute of Robotics and System Dynamics \\ D-82234 Wessling, Germany. \\ Fax: +(49)-8153-28-1441, E-mail: andras.varga@dlr.de
}

Keywords : linear systems; inner-outer factorization; numerical methods; descriptor realizations.

\begin{abstract}
In this paper we give a theoretical and a computational solution to the most general inner-outer factorization problem formulated for a discrete-time system $G$. Our method is based on descriptor state-space computations and relies on an efficient dislocation of the minimal indices and of the "unstable" zeros of $G$ by left multiplication with all-pass factors. The minimal indices are dislocated by solving for the stabilizing solution an algebraic Riccati equation of order $n_{\ell}$ (the sum of left minimal indices) while the $n_{b}$ unstable zeros are dislocated by solving a Lyapunov equation of order $n_{b}$. The results reported here are a nontrivial extension of a recently developed approach to the continuous-time inner-outer factorization problem.
\end{abstract}

\section{Introduction}

Throughout the paper $\mathbb{D}, \mathbb{C}, \overline{\mathbb{D}}, \overline{\mathbb{C}}$ stand for the unit disk, the complex plane, and their closures, respectively. The general discrete-time inner-outer factorization problem we deal with is formulated as follows.

Inner-Outer Factorization Problem. Given $G(z)$, an arbitrary real rational matrix analytic in $\overline{\mathbb{C}} \backslash \mathbb{D}$ (i.e., proper and stable in discrete-time), determine two real rational matrices $G_{i}(z)$ and $G_{o}(z)$ such that

$$
G(z)=G_{i}(z) G_{o}(z),
$$

$G_{i}(z)$ and $G_{o}(z)$ are both analytic in $\overline{\mathbb{C}} \backslash \mathbb{D}, G_{i}^{*}(z) G_{i}(z)=$ $I$, and $G_{o}(z)$ has a right inverse analytic in $\overline{\mathbb{C}} \backslash \overline{\mathbb{D}}$. $G_{i}(z)$ is called an inner factor, $G_{o}(z)$ is called an outer factor, and (1) defines an inner-outer factorization of $G(z)$.
The methods developed in this paper are applicable for a more general class of factorizations (1), in which $G$ can be arbitrary (improper/unstable), and $G_{i}$ is required to be inner and $G_{o}$ to have a right inverse analytic in $\overline{\mathbb{C}} \backslash \overline{\mathbb{D}}$.

Inner-outer factorizations appear throughout in control systems, identification, signal processing, and circuit theory, and it is surely hopeless to give here a short but still comprehensive account on all applications in which they occur. For a historical perspective we refer the interested reader to [6]. However, all of the approaches proposed so far stick on some simplifying assumptions on $G$ which are inherent to the respective methods (see [2], [13], [5]). We limit our discussion to only two of recently proposed methods.

One of the most general method available so far for discrete-time systems can deal with $G$ of arbitrary rank but without zeros on the unit circle [3], [4]. However, if such zeros are present, semi-stabilizing (or weak stabilizing) solutions instead of stabilizing solutions to Riccati equations have to be computed and this implies that for the associated symplectic pencil a symmetric separation of the eigenvalues on the unit circle must be determined. Unfortunately, no numerical method to cope efficiently with this task is available.

Recently, the most general inner-outer and spectral factorizations for continuous-time systems have been solved in [6] by using a method of zeros/minimal indices dislocation with all-pass factors. In this paper we extend this method to the class of discrete-time systems. The extension is not trivial, since in discrete-time improper all-pass factors are needed for the dislocation, and appropriate generalized (descriptor) state-space representations have to be used. 


\subsection{Outline of the proposed approach}

Let $G(z)$ be an arbitrary $p \times m$ real rational matrix of rank $r$. Our approach to the inner-outer factorization problem relies on two basic factorizations.

\section{Step 1. Row compression by all-pass factors}

We factorize an arbitrary $G(z)$ as

$$
G(z):=G_{a}(z) \widetilde{G}(z),
$$

where $G_{a}(z)$ is all-pass with all poles in $\mathbb{D}$, and $\widetilde{G}(z)$ is row compressed, i.e., the trailing $p-r$ rows of $\widetilde{G}(z)$ are zero

$$
\left.\widetilde{G}(z)=\left[\begin{array}{c}
\widetilde{G}_{1}(z) \\
O
\end{array}\right]\right\} \begin{aligned}
& \} r \\
& \} p-r
\end{aligned} .
$$

This amounts to dislocating all minimal indices to the left (modifying them such that all become zero), and introducing instead zeros in $\mathbb{D}$. We chose $G_{a}(z)$ to have the smallest possible McMillan degree $n_{\ell}$ which is equal to the sum of all left minimal indices of $G(z)$. The computation of $G_{a}(z)$ involves the solution for the stabilizing solution of a standard Riccati equation of order $n_{\ell}$. Combining (3) and (2) we get

$$
G(z)=G_{i 1}(z) \widetilde{G}_{1}(z)
$$

where $G_{a}(z)=\left[\begin{array}{ll}G_{i 1}(z) & G_{i 2}(z)\end{array}\right], G_{i 1}(z)$ is inner, $\widetilde{G}_{1}(z)$ is surjective and has the same zeros in $\overline{\mathbb{C}} \backslash \mathbb{D}$ as $G(z)$, while its zeros in $\mathbb{D}$ are the union of the zeros in $\mathbb{D}$ of $\widetilde{G}(z)$ with the zeros of $G_{a}^{-1}(z)$.

\section{Step 2. Dislocation of zeros by all-pass factors}

We factorize a surjective $G(z)$ as

$$
G(z)=G_{a}(z) \widetilde{G}(z)
$$

where $G_{a}(z)$ is inner and $\widetilde{G}(z)$ is surjective and has no zeros in $\overline{\mathbb{C}} \backslash \overline{\mathbb{D}}$ (i.e., $\widetilde{G}(z)$ is outer). This amounts to dislocating all zeros in $\overline{\mathbb{C}} \backslash \overline{\mathbb{D}}$ of $G(z)$ and reflecting them into symmetric positions in $\mathbb{D}$ with respect to the unit circle. Again, we chose $G_{a}(z)$ to have the smallest possible McMillan degree which is equal to the number $n_{b}$ of zeros of $G(z)$ in $\overline{\mathbb{C}} \backslash \overline{\mathbb{D}}$. The computation of $G_{a}(z)$ is achieved by solving a Lyapunov equation of order $n_{b}$.

These above two steps are then performed successively to get the inner-outer factorization as explained in Section 5. Notice that although the inner-outer factorization is defined for a stable $G$, Step 1 works for arbitrary $G$ and Step 2 works for any surjective $G$ (not necessarily stable).

The paper is organized as follows. In Section 2 we recall some basic facts on rational matrices and descriptor realizations. In Section 3 we give two special orthogonal decompositions of the system pencil which exhibit the relevant Kronecker structure. These two spectral decompositions correspond to the two steps of the factorization process and are the key of our approach. Section 4 contains the main results of the paper. Here we present the two basic factorizations described above and for each factorization we provide explicit state-space formulas for the factors. We discuss the inner-outer factorization in Section 5. We summarize the main aspects of the proposed approach by some conclusions. Due to space limitations all the proofs are omitted.

\section{Preliminaries}

Let $G(z)$ be an arbitrary (possibly improper) real rational matrix. Throughout the paper we use the following notation for the structural elements of $G(z): r$ stands for the rank over rational matrices, $n$ denotes the McMillan degree (which equals the number of poles counting multiplicities and including infinity), $n_{z}=n_{b}+n_{g}$ is the number of zeros (counting multiplicities and including infinity), where $n_{g}$ is the number of "good" zeros in $\Gamma_{g}:=\overline{\mathbb{D}}$ and $n_{b}$ is the number of "bad" zeros in $\Gamma_{b}:=\overline{\mathbb{C}}-\overline{\mathbb{D}}, \mathcal{Z}(G(s))$ denotes the union of zeros (with multiplicities), $n_{\ell}$ is the sum of degrees of any minimal basis of the left null space (these degrees are called left minimal indices), $n_{r}$ is the sum of degrees of any minimal basis of the right null space (these degrees are called right minimal indices). For precise definitions of all these structural elements see [1], [8], [11]. For a rational matrix there is an interesting relation [11] among its structural elements of the form

$$
n=n_{z}+n_{r}+n_{\ell}
$$

The relation (5) will be insightfull for the problems treated in the sequel.

It is well known that any rational matrix $G$ (even improper or polynomial) has a descriptor realization

$$
G(z)=\left[\begin{array}{c|c}
A-z E & B \\
\hline C & D
\end{array}\right]:=C(z E-A)^{-1} B+D,
$$

where the so called pole pencil $A-z E$ is regular, i.e., it is square and $(\operatorname{det}(A-z E) \not \equiv 0)$, and all the intervening matrices in (6) have real coefficients. By $\Lambda(A-z E)$ we shall denote the union of generalized eigenvalues of an arbitrary (possibly singular) pencil $A-z E$ (finite and infinite, multiplicities counting). The descriptor representation (6) of $G$ is called minimal if the dimension $k$ of the square matrices $E$ and $A$ is as small as possible. With a particular realization (6) of $G(z)$ we associate also the system pencil

$$
\mathcal{S}_{G}(z)=\left[\begin{array}{cc}
A-z E & B \\
C & D
\end{array}\right] .
$$

The pole pencil and the system pencil play a fundamental role as their Weierstrass and Kronecker canonical forms (see [9]), respectively, are intimately related to the structural elements of the rational matrix as described in [8] and [11]. 
The principal drawback of realizations of the form (6) is that their minimal possible order is greater than the McMillan degree of $G$, unless $G$ is proper, and this brings important technical difficulties in factorization problems in which the McMillan degree plays a paramount role. A remedy to this is to use a generalization of (6) in which either the " $B$ " or the " $C$ " matrix is replaced by a matrix pencil, as explained further.

Any rational matrix $G$ has a realization of the form

$$
\begin{aligned}
G(z) & =\left[\begin{array}{c|c}
A-z E & B-z F \\
\hline C & D
\end{array}\right] \\
& :=C(z E-A)^{-1}(B-z F)+D,
\end{aligned}
$$

and for any fixed real numbers $\alpha$ and $\beta$, not both zero, there also exists a realization of the form

$$
\begin{aligned}
G(z) & =\left[\begin{array}{c|c}
A-z E & B(\alpha-z \beta) \\
\hline C & D
\end{array}\right] \\
& :=C(z E-A)^{-1} B(\alpha-z \beta)+D
\end{aligned}
$$

where in both (8) and (9) the pole pencil $A-z E$ is regular. A realization (9) will be called centered at $\frac{\alpha}{\beta}$. If $\beta=0$ then we have a realization centered at infinity.

We call realizations of the type (8) or (9) minimal if the dimension of the square matrices $A$ and $E$ (also called the order of the realization) is as small as possible among all realizations of the respective kind. It can be easily shown that any rational matrix $G(z)$ has a minimal realization (8) of order equal to $\delta(G)$ (the McMillan degree of $G$ ). For any fixed $\alpha$ and $\beta$, not both zero, and such that $\frac{\alpha}{\beta}$ is not a pole of $G$ there exists a minimal realization (9) of order equal to $\delta(G)$. An extensive discussion of realizations (9) is given in [7].

\section{Spectral decompositions of the system pencil}

In this section we give two spectral decompositions of the system pencil $\mathcal{S}_{G}(z)$ which have a key role in establishing the conditions of dislocating the left minimal indices and the zeros, respectively. We start with a spectral decomposition that outlines in an appropriate form the left Kronecker structure of $\mathcal{S}_{G}(z)$.

Theorem 3.1 Let $G(z)$ be a $p \times m$ real rational matrix of McMillan degree $n$, of rank $r$, having $n_{z}$ zeros, and the sums of minimal indices to the left and right $n_{\ell}$ and $n_{r}$, respectively. Then there exists a $k$-dimensional minimal realization (6) of $G(z)$ and two orthogonal matrices $Q$ and $Z$ such that

$$
\left[\begin{array}{cc}
I & O \\
O & Q^{T}
\end{array}\right]\left[\begin{array}{cc}
A-z E & B \\
\hdashline & D
\end{array}\right] Z=
$$

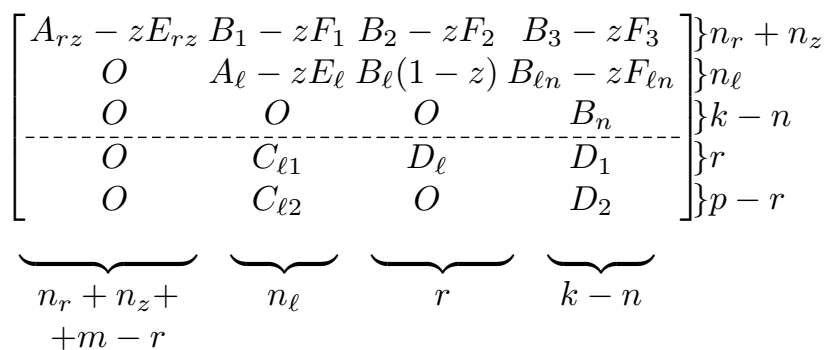

where

(a) $\mathcal{Z}(G(z))=\Lambda\left(A_{r z}-z E_{r z}\right)$ and $A_{r z}-z E_{r z}$ is surjective for all $z \notin \mathcal{Z}(G(z))$.

(b) $A_{\ell}-E_{\ell}, D_{\ell}$ and $B_{n}$ are invertible and (bi) $\operatorname{rank}\left[\begin{array}{cc}A_{\ell}-z E_{\ell} & B_{\ell}(1-z)\end{array}\right]=n_{\ell}, \forall z \in \mathbb{C}$;

(bii) $\operatorname{rank}\left[\begin{array}{c}A_{\ell}-z E_{\ell}-B_{\ell} D_{\ell}^{-1} C_{\ell 1}(1-z) \\ C_{\ell 2}\end{array}\right]=n_{\ell}$, $\forall z \in \mathbb{C}$.

Moreover, the minimal realization and the orthogonal matrices $Q$ and $Z$ can be effectively constructed by a finite algorithm (omitted here for brevity).

We assume now that $G(z)$ has no left minimal indices, i.e., it is surjective. As we shall see further, this is always possible after we have dislocated the minimal indices. The next theorem is key to understand the conditions for dislocating the zeros of $G(z)$ (both finite and infinite).

Theorem 3.2 Let $G(z)$ be a $p \times m$ real rational matrix of McMillan degree $n$, of rank $p$ (i.e., $G(z)$ is surjective), having $n_{z}=n_{b}+n_{g}$ zeros, where $n_{b}$ and $n_{g}$ are the number of zeros in $\Gamma_{b}$ and $\Gamma_{g}$, respectively, and the sum of minimal indices to the right $n_{r}$. Then there exists a $k$ dimensional minimal realization $(6)$ of $G(z)$ and an orthogonal matrix $Z$ such that

$$
\left[\begin{array}{cc}
A-z E & B \\
\hdashline C & D
\end{array}\right] Z=
$$

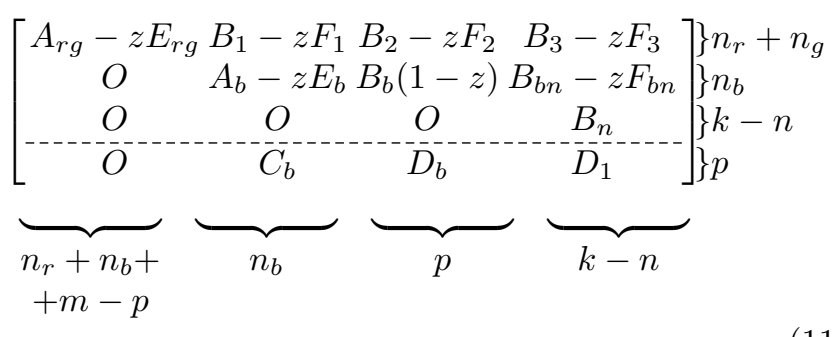

where

(a) $\mathcal{Z}(G(z)) \cap \Gamma_{g}=\Lambda\left(A_{r g}-z E_{r g}\right)$ and $A_{r g}-z E_{r g}$ is surjective for all $z \notin \mathcal{Z}(G(z)) \cap \Gamma_{g}$.

(b) $A_{b}-E_{b}, D_{b}, B_{n}$ are invertible and (bi) $\Lambda\left(A_{b}-z E_{b}-B_{b} D_{b}^{-1} C_{b}(1-z)\right)=\mathcal{Z}(G(z)) \cap \Gamma_{b}$, (bii) $\operatorname{rank}\left[\begin{array}{cc}A_{b}-z E_{b} & B_{b}(1-z)\end{array}\right]=n_{b}, \forall z \in \mathbb{C}$.

Moreover, the minimal realization and the orthogonal matrix $Z$ can be effectively constructed by a finite algorithm (omitted here for brevity). 


\section{The basic factorization steps}

\subsection{Row compression by all-pass factors}

Theorem 4.1 Let $G(z)$ be a real rational matrix and let (6) be a minimal realization of $G(z)$, and $Q$ and $Z$ orthogonal matrices as in Theorem 3.1 such that (10) holds. Then we have:

1. The algebraic Riccati equation

$$
\begin{aligned}
& E_{\ell}^{T} X E_{\ell}-A_{\ell}^{T} X A_{\ell}-\left[\left(E_{\ell}-A_{\ell}\right)^{T} X B_{\ell}+C_{\ell 1}^{T} D_{\ell}\right) \\
& \times\left(D_{\ell}^{T} D_{\ell}\right)^{-1}\left(B_{\ell}^{T} X_{s}\left(E_{\ell}-A_{\ell}\right)+D_{\ell}^{T} C_{\ell 1}\right]+C_{\ell}^{T} C_{\ell}=0
\end{aligned}
$$

has a stabilizing invertible solution $X_{s}$ such that $\Lambda\left(A_{\ell}-\right.$ $\left.z E_{\ell}+B_{\ell} F_{s}(1-z)\right) \subset \mathbb{D}$, where

$$
F_{s}:=-\left(D_{\ell}^{T} D_{\ell}\right)^{-1}\left(B_{\ell}^{T} X\left(E_{\ell}-A_{\ell}\right)+D_{\ell}^{T} C_{\ell 1}\right)
$$

$\begin{array}{ll}\text { is } \quad \text { the } & \text { stabilizing Riccati feedback and } \\ C_{\ell}^{T}:=\left[\begin{array}{ll}C_{\ell 1}^{T} & C_{\ell 2}^{T}\end{array}\right] \text {. } & \end{array}$

2. Let

$$
\begin{aligned}
& G_{i}(z)=\left[\begin{array}{ll}
G_{i 1}(z) & G_{i 2}(z)
\end{array}\right] \\
& =Q\left[\begin{array}{c|c}
A_{\ell}-z E_{\ell}+B_{\ell} F_{s}(1-z) & B_{\ell} D_{\ell}^{-1}(1-z) \\
\hline C_{\ell 1}+D_{\ell} F_{s} & I \\
C_{\ell 2} & O
\end{array}\right. \\
& \left.\frac{-X_{s}^{-1}\left(E_{\ell}-A_{\ell}\right)^{-T} C_{\ell 2}^{T}(1-z)}{O}\right]
\end{aligned}
$$

and

$$
G_{o}(z)=\left[\begin{array}{c}
G_{o 1}(z) \\
O
\end{array}\right]=\left[\begin{array}{c|c}
A-z E & B \\
\hline D_{\ell} H_{1} & D_{\ell} H_{2} \\
O & O
\end{array}\right]=
$$

where

$$
\left[\begin{array}{ll}
H_{1} & H_{2}
\end{array}\right]:=\left[\begin{array}{llll}
O & -F_{s} & I & O
\end{array}\right] Z^{T}
$$

and $p(z)=(1-z)$. Then $G_{i}(z)$ is a $p \times p$ inner matrix, the realization (14) is minimal, $G(z)=G_{i}(z) G_{o}(z), G_{o 1}(z)$ has no left minimal indices, and $\mathcal{Z}\left(G_{o}(z)\right)$ has $n_{b}$ elements in $\overline{\mathbb{C}}-\overline{\mathbb{D}}$.

We switch now to the problem of dislocating zeros.

\subsection{Zeros dislocation by all-pass factors}

Theorem 4.2 Let $G(z)$ be a surjective real rational matrix with the minimal minimal realization (6) and let $Z$ be an orthogonal matrix as in Theorem 3.2 satisfying (11). Then:

1. The Lyapunov equation

$$
\begin{aligned}
& \left(E_{b}+A_{b}-2 B_{b} D_{b}^{-1} C_{b}\right) Y\left(E_{b}-A_{b}\right)^{T}+\left(E_{b}-A_{b}\right) Y \\
& \times\left(E_{b}+A_{b}-2 B_{b} D_{b}^{-1} C_{b}\right)^{T}-2 B_{b}\left(D_{b}^{T} D_{b}\right)^{-1} B_{b}^{T}=0
\end{aligned}
$$

has a unique invertible solution such that $\Lambda\left(A_{b}-z E_{b}+\right.$ $\left.B_{b} F_{s}(1-z)\right) \subset \mathbb{D}$, where

$$
F_{s}:=-\left(D_{b}^{T} D_{b}\right)^{-1}\left(B_{b}^{T}\left(E_{b}-A_{b}\right)^{-T} Y^{-1}+D_{b}^{T} C_{b}\right) .
$$

2. Let

$G_{i}(s)=\left[\begin{array}{c|c}A_{b}-z E_{b}+B_{b} F_{s}(1-z) & B_{b} D_{b}^{-1}(1-z) \\ \hline C_{b}+D_{b} F_{s} & I\end{array}\right]$

and

$$
G_{o}(s)=\left[\begin{array}{c|c}
A-z E & B \\
\hline D_{b} H_{1} & D_{b} H_{2}
\end{array}\right]
$$

where

$$
\left[\begin{array}{ll}
H_{1} & H_{2}
\end{array}\right]:=\left[\begin{array}{llll}
O & F_{s} & I & O
\end{array}\right] Z^{T} .
$$

Then $G_{i}(z)$ is square inner, the realization (19) is minimal, $G(z)=G_{i}(z) G_{o}(z)$, and $\mathcal{Z}\left(G_{o}\right) \subset \overline{\mathbb{D}}$.

\section{Solution to the factorization problems}

In this section we explain briefly how we can apply the already obtained results to compute the inner-outer factorization in the most general setting.

Let $G(z)$ be an arbitrary stable rational matrix (analytic in $\overline{\mathbb{C}} \backslash \mathbb{D})$.

Step 1. Use Theorem 4.1 to determine a factorization

$$
G(z)=G_{i}^{(1)}(z) G_{o}^{(1)}(z)
$$

where

$$
\begin{gathered}
G_{i}^{(1)}(z)=\left[G_{i 1}^{(1)}(z) \quad G_{i 2}^{(1)}(z)\right] \\
G_{o}^{(1)}(z)=\left[\begin{array}{c}
G_{o 1}^{(1)}(z) \\
O
\end{array}\right] .
\end{gathered}
$$

The resulting $G_{i}^{(1)}(z)$ has all poles in $\mathbb{D}$ and it is inner and square, while $G_{o 1}^{(1)}(z)$ is surjective with $n_{b}$ zeros in $\overline{\mathbb{C}} \backslash \overline{\mathbb{D}}$.

Step 2. Use Theorem 4.2 to determine a factorization

$$
G_{o}^{(1)}(z)=G_{i}^{(2)}(z) G_{o}^{(2)}(z)
$$

where $G_{i}^{(2)}(z)$ has all poles in $\mathbb{D}$ and it is inner (and square), while $G_{O}^{(2)}(z)$ is surjective with all zeros in $\overline{\mathrm{D}}$ (i.e., it is outer).

Result. The inner-outer factorization results as

$$
G(z)=G_{i}(z) G_{o}(z)
$$

where

$$
\begin{gathered}
G_{i}(z):=G_{i 1}^{(1)}(z) G_{i}^{(2)}(z), \\
G_{o}(z):=G_{o}^{(2)}(z) .
\end{gathered}
$$




\section{Conclusions}

In this paper we have given a complete solution to the inner-outer factorization problem formulated for a discrete-time system in the most general setting possible. We have provided both theoretical solutions and numerically reliable procedures.

All computational steps of the proposed procedures can be performed by using exclusively numerically-sound algorithms involving orthogonal transformations to the largest extent possible. The procedures are well suited for robust and modular software implementation and the resulting algorithms are computationally efficient. In all cases the methods are exactly tailored to the dimension of the problem to be solved avoiding unnecessary redundancy. As an example, the solution of descriptor Riccati equations of order $n$ that are usually employed is generally avoided. The use of the numerically intractable "weak" stabilizing solution of Riccati equations in case of unit circle zeros is also completely avoided. Instead, we solve a reduced order standard Riccati and a Lyapunov equation for which numerical software is already available.

We may apply the methods of this paper to obtain the solutions to the spectral factorization problem formulated for a discrete-time system. Again, the same level of generality may be achieved.

\section{Acknowledgment}

This research has been made possible by a Fellowship of the Alexander von Humboldt Foundation, Bonn.

\section{References}

[1] Forney G. D., "Minimal bases of rational vector spaces, with applications to multivariable linear systems", SIAM J. of Control, 13, 493-520, (1975).

[2] Gu D. W., Tsai M. C., O'Young S. D., Postlethwaite I, "State-space formulae for discrete-time $H_{\infty}$ optimization" Int. J. Control, 49, 1683-1723, (1989).

[3] Ionescu V., Oară C., "Spectral and inner-outer factorizations for discrete-time systems" IEEE Trans. Autom. Control, 41, 1840-1845, (1996).

[4] Ionescu V., Oară C., Weiss M., Generalized Riccati Theory and Robust Control: A Popov Function Approach, John Wiley \& Sons, New York, (1999), ISBN 0-471-97147-2.

[5] Katayama T., "A spectral factorization algorithm for discrete-time descriptor systems via generalized eigenproblems" Lin. Alg. \& Appl., 205-206, 869891, (1994).
[6] Oară C., Varga A., "Computation of general innerouter and spectral factorizations", to appear in IEEE Trans. Auto. Contr..

[7] Oară C., "Minimal factorization of rational matrices: the general case", to appear in Proceedings of MTNS 1998, Padova, Italy, 6-10 July 1998.

[8] Rosenbrock H. H., State-Space and Multivariable Theory, Wiley, New York, (1970).

[9] Van Dooren P., "The computation of Kronecker's canonical form of a singular pencil", Lin. Alg. \& Appl., 27, 103-141, (1979).

[10] Van Dooren P., "Rational and polynomial matrix factorizations via recursive pole-zero cancelation", Lin. Alg. \& Appl., 137/138, 663-697, (1990).

[11] Verghese G., Van Dooren P., Kailath T., "Properties of the system matrix of a generalized state-space system", Int. J. Control, 30, 235-243, (1979).

[12] Verghese G., Lévy B., Kailath T., "A generalized state-space for singular systems", IEEE Trans. Autom. Control, 26, 811-831, (1981).

[13] Zhang Z., Freudenberg J. S., "Formulas for minimum-phase/all-pass factorization of discretetime transfer functions", Control-Theory and Advanced Technology, 8, 743-754, (1992). 